\section{Variation in Lutein, $\beta$-carotene, and Chlorophyll Concentrations among Brassica oleracea Cultigens and Seasons}

\author{
Dean A. Kopsell ${ }^{1}$, David E. Kopsell ${ }^{2}$, and Mark G. Lefsrud ${ }^{3}$ \\ Department of Plant Biology, The University of New Hampshire, Durham, \\ $\mathrm{NH} 03824$ \\ Joanne Curran-Celentano ${ }^{4}$ and Laura E. Dukach ${ }^{5}$ \\ Department of Animal and Nutritional Sciences, The University of New \\ Hampshire, Durham, NH 03824
}

Additional index words. carotenoids, collards, germplasm, HPLC, human health, kale

\begin{abstract}
Green leafy vegetables are important sources of dietary carotenoids, and members of Brassica oleracea $\mathrm{L}$. var. acephala rank highest for reported levels of lutein and $\beta$-carotene. Twenty-three leafy $\boldsymbol{B}$. oleracea cultigens were field grown under similar fertility over two separate years and evaluated for leaf lutein and $\beta$-carotene accumulation. Choice of $B$. oleracea cultigen and year significantly affected carotenoid levels. Lutein concentrations ranged from a high of $13.43 \mathrm{mg}$ per $100 \mathrm{~g}$ fresh weight (FW) for B. oleracea var. acephala 'Toscano' to a low of $4.84 \mathrm{mg} / 100 \mathrm{~g} \mathrm{FW}$ for B. oleracea var. acephala 343-93G1. $\beta$-carotene accumulations ranged from a high of $10.00 \mathrm{mg} / 100 \mathrm{~g} \mathrm{FW}$ for $B$. oleracea var. acephala 'Toscano' to a low of $3.82 \mathrm{mg} / 100 \mathrm{~g}$ FW for B. oleracea var. acephala 3034393G1. Carotenoid concentrations were significantly higher in year 2 than in year 1, but rank order among the cultigens for both lutein and B-carotene did not change between the years. During each year, there were high correlations between leaf carotenoid and chlorophyll pigments. Under similar growing conditions, choice of $B$. olerace $a$ cultigen will influence carotenoid accumulation, and this may affect the health benefits of consuming these leafy green vegetable crops.
\end{abstract}

Carotenoids are secondary plant compounds that form lipid soluble yellow, orange, and red pigments. Lutein $\left(3 R, 3^{\prime} R, 6^{\prime} R \beta, \varepsilon-\right.$ carotene-3,3'diol), a oxygenated xanthophyll, and $\beta$-carotene ( $\beta, \beta$-carotene), a hydrocarbon carotene, are examples of two nutritionally important plant-derived carotenoids (Zaripheh and Erdman, Jr., 2002). In green plants, carotenoids are bound to specific protein complexes of PSI and PSII, and along with chlorophyll $a$ and $b$, function in light harvesting. Carotenoids also protect photosynthetic structures by quenching ${ }^{3} \mathrm{Chl}$ and ${ }^{1} \mathrm{O}_{2}$ to inhibit oxidative damage (Tracewell et al., 2001).

Kale (Brassica oleracea L. var. acephala) ranks highest among all leafy vegetable crops for lutein and $\beta$-carotene content (Sommerburg et al., 1998). Lutein/zeaxanthin levels within leafy $B$. oleracea cultigens are reported to

Received for publication 11 June 2003. Accepted for publication 4 Dec. 2003. This paper was funded in part by a grant received by the Cooperative State Research, Education, and Extension Service, U.S. Dept. of Ag riculture, under Agreement No. 2001-52102-11254. This is Scientific Contribution No. 2207 from the New HampshireAgricultural Experiment Station. The authors wish to thank the Northeastern Regional PI Station, USDA-ARS Plant Genetic Resources Unit, Cornell University, Geneva, N.Y., for providing seed used in these experiments and John McLean, Evan Ford, and the Woodman Horticultural Research Farm at UNH for their technical support of this research.

${ }^{1}$ Assistant Professor; to whom reprint requests should be addressed. E-mail: kopsell@ cisunix.unh.edu

${ }^{2}$ Postdoctoral Research Scientist.

${ }^{3}$ Graduate Research Assistant.

${ }^{4}$ Associate Professor.

${ }^{5}$ Research Technician. range from 8.0 to $39.5 \mathrm{mg} / 100 \mathrm{~g}$ fresh tissue, while levels of $\beta$-carotene range from 2.8 to $14.5 \mathrm{mg} / 100 \mathrm{~g}$ fresh tissue (Sommerburg et al., 1998; Khachik et al., 1986). However, Kachick et al. (1986) admitted the upper end of the reported lutein and $\beta$-carotene ranges were obtained from one homogenous sample per cultigen, and would likely fail to be representative of material consumed nationwide in the United States.

The content and distribution of carotenoids in plants appears to be shaped by physiological, genetic, and biochemical factors (Goldman et al., 1999; Grusak et al., 1999). Mercadante and Rodriguez-Amaya (1991) observed differences in lutein and $\beta$-carotene content of two field-grown kale cultivars, 'Manteiga' and 'Tronchuda', in Brazil. Seasonal variation between winter and summer production in lutein and $\beta$-carotene levels were also observed between the two cultivars. Kurilich et al. (1999) found significant differences in $\beta$-carotene and $\alpha$-tocopherol content within and among 50 broccoli (B. oleracea L. var. italica) accessions. Klein and Perry (1982) reported significant differences in carotenoid (vitamin A) content of five different vegetables \{carrot (Daucus carota L.), celery [Apium graveolens L. var. dulce (Mill.) Pers.], tomato (Lycopersicon esculentum Mill.), cabbage (Brassica oleracea L. Capitata Group), and corn (Zea mays L.) \} purchased from six different U.S. locations, but could not conclude if differences were due to cultivar, culture, or postharvest handling procedures.

The nutritional and medicinal importance of dietary carotenoid consumption is being established (Balentine et al., 1999). Dietary intake of lutein, $\beta$-carotene, and $\alpha$-carotene has been associated with reduced risk of lung cancer in both men and women (Le Marchand et al., 1993). Diets containing carotenoid-rich fruits and vegetables are associated with decreased risk of chronic eye diseases, including cataract and age-related macular degeneration (Johnson et al., 2000). Although direct evidence of carotenoid antioxidant and photoprotective functions in humans is lacking, dietary intake of carotenoids is still recommended (Krinsky, 2002; Olsen, 1999).

Because of the reported health benefits of consuming carotenoid-rich, green leafy vegetables, the objective of this study was to characterize the variability of lutein and $\beta$-carotene accumulation in 23 different $B$. olerace $a$ cultigens suitable for production in the northeastern region of the United States. Due to previous reports of seasonal variability of carotenoid accumulation, cultigens were evaluated over two separate growing seasons.

\section{Materials and Methods}

Twenty-three B. oleracea cultigens, including commercial cultivars and USDA-ARS accessions, were seeded into artificial media (Pro-mix BX; Premier Horticulture, Dorval, Quebec; Table 1) on 16 June 2001 and 18 June 2002. The medium was supplied with bottom heat $\left(23^{\circ} \mathrm{C}\right)$ and plants were greenhouse grown $\left(22^{\circ} \mathrm{C}\right.$ day $/ 14^{\circ} \mathrm{C}$ night set points) for 4 weeks under natural photoperiods (lat. $43^{\circ} 09^{\prime} \mathrm{N}$ ). Nutrients were applied as needed using 200 $\mathrm{mg} \cdot \mathrm{L}^{-1}$ Peter's 20N-6.9P-16.6K water-soluble fertilizer (Grace-Sierra, Milpitas, Calif.).

Plants were transplanted into the field on or about the second week of July during both years. Fertilizer was applied 1 week prior to transplanting and cultigens were grown according to New England Cooperative Extension guidelines for minor cole crops (Howell et al., 2002). Each cultigen was planted in a plot consisting of two rows of 12 plants each at the recommended spacing of $45 \mathrm{~cm}$ within rows and $91 \mathrm{~cm}$ between rows. Plots were replicated three times in a randomized complete-block design for each year of evaluation. Irrigation was supplied during plant growth to ensure plants received a total of $2.54 \mathrm{~cm}$ of water per week. Nitrogen was sidedressed as $\mathrm{NH}_{4} \mathrm{NO}_{3}$ at the rate of $67.2 \mathrm{~kg} \cdot \mathrm{ha}^{-1} 3$ weeks after transplanting. Plants were harvested on 25 Aug. 2001 and 27 Aug. 2002. From 15 July to 27 Aug. 2001, the average daily temperature was $19.5^{\circ} \mathrm{C}$ (with $6 \mathrm{~d}$ reaching temperatures $>32.0$ ${ }^{\circ} \mathrm{C}$ ); average daily photosynthetically active radiation $(P A R)$ was $558 \mu \mathrm{mol} \cdot \mathrm{m}^{-2} \cdot \mathrm{s}^{-1}$, and total rainfall was $5.61 \mathrm{~cm}$. From 15 July to $27 \mathrm{Aug}$. 2002 , the average daily temperature was 22.3 ${ }^{\circ} \mathrm{C}$ (with $12 \mathrm{~d}$ reaching temperatures $>32.0$ ${ }^{\circ} \mathrm{C}$ ), average daily $P A R$ was $584 \mu \mathrm{mol} \cdot \mathrm{m}^{-2} \cdot \mathrm{s}^{-1}$, and total rainfall was $4.95 \mathrm{~cm}$ (Univ. of New Hampshire weather station, Durham). At harvest, the third most fully expanded leaf from 10 uniform plants per replicate was removed and combined for carotenoid analysis (Jones, 1972). Tissues were lyophilized for $48 \mathrm{~h}$ (model 
Breeding, Cultivars, Rootstocks, \& Germplasm Resources

6 L FreeZone; LabConCo, Kansas City, Mo.) and stored at $-80^{\circ} \mathrm{C}$ prior to extraction.

Plant pigments were extracted from freezedried tissue according to the method of Beecher and Howard (USDAFood Composition Laboratory, Beltsville, Md.; personal communications), which is a modification of the method of Khachik et al. (1986). A 0.10-g subsample was rehydrated with $0.8 \mathrm{~mL}$ of $\mathrm{ddH}_{2} \mathrm{O}$ at 40 ${ }^{\circ} \mathrm{C}$ for $20 \mathrm{~min}$. After incubation, $0.8 \mathrm{~mL}$ of the internal standard ethyl- $\beta$-8-apo-carotenoate (Sigma Chemical Co., St. Louis) and $2.5 \mathrm{~mL}$ of tetrahydrofuran (THF) stabilized with 25 $\mathrm{mg} \cdot \mathrm{L}^{-1}$ 2,6-Di-tert-butyl-4-methoxyphenol (BHT) were added. The sample was homogenized in a Potter-Elvehjem (Kontes, Vineland, N.J.) tissue grinding tube using $\approx 25$ insertions with a pestle attached to a drill press (model Craftsman 15 inch Drill Press; Sears, Roebuck and Co., Hoffman Estates, Ill.) set at $540 \mathrm{rpm}$. During homogenation, the tube was immersed in ice to dissipate heat. The tube was then placed into a clinical centrifuge for $3 \mathrm{~min}$ at $500 g_{\mathrm{n}}$. The supernatant was removed and the sample pellet was resuspended in $2.0 \mathrm{~mL}$ THF and homogenized again with the same extraction technique. The extraction procedure was repeated two more times to obtain a colorless supernatant. The combined supernatants were reduced to $0.5 \mathrm{~mL}$ using nitrogen (model $\mathrm{N}$ EVAP 111; Organomation Inc., Berlin, Mass.) at $40{ }^{\circ} \mathrm{C}$ and $2.5 \mathrm{~mL} \mathrm{MeOH}$ and $2.0 \mathrm{~mL}$ THF were added to the sample prior to HPLC analysis. Saponification was not performed on the B. oleracea samples because it has been found to degrade carotenoids and reduce recovery (personal observation; Kimura et al., 1990; Mercadante and Rodriguez-Amaya, 1991).

An Agilent 1100 series HPLC unit with a photo diode array detector (Agilent Technologies, Palo Alto, Calif.) was used for sample separation. All samples were analyzed using a RP C- $18,80 \AA$, $3.0 \mu \mathrm{m}, 300 \times 4.6-\mathrm{mm}$ column (Adsorbosphere HS; Alltech, Deerfield, Ill.) fitted with a $7.5 \times 4.0-\mathrm{mm} 5.0-\mu \mathrm{m}$ guard column (All Guard C-18; Alltech). The column was maintained at $16{ }^{\circ} \mathrm{C}$ using a thermostatted column compartment. Eluents were: (A) $75 \%$ acetonitrile, $20 \%$ methanol, $5 \%$ hexane, $0.05 \%$ BHT, and $0.013 \%$ triethylamine (TEA) in water (v/v); and (B) $50 \%$ acetonitrile, $25 \%$ THF, $25 \%$ hexane, and $0.013 \%$ TEA in water $(\mathrm{v} / \mathrm{v})$. The flow rate was $0.7 \mathrm{~mL} \cdot \mathrm{min}^{-1}$ and the gradient is $100 \%$ A for $30 \mathrm{~min}, 50 \%$ A and $50 \%$ $\mathrm{B}$ for $2 \mathrm{~min}$; $100 \% \mathrm{~B}$ for $2 \mathrm{~min}$; and $50 \% \mathrm{~A}$ and $50 \% \mathrm{~B}$ for $2 \mathrm{~min}$. The eluent composition was returned to $100 \% \mathrm{~A}$ and the column was equilibrated for $10 \mathrm{~min}$ prior to the next injection. Eluted compounds from a $20-\mu$ Linjection were detected at 452,652 , and $665 \mathrm{~nm}$ and data sets were collected, recorded, and integrated using 1100 HPLC ChemStation Software (Agilent Technologies). Peak assignment was performed by comparing retention times and line spectra obtained from photodiode array detection with authentic standards (lutein from Carotenature, Lupsingen, Switzerland; $\beta$-carotene, chlorophyll $a$, and chlorophyll $b$ from Sigma Aldrich, St. Louis).

Data sets were analyzed by the GLM procedures of SAS (Cary, N.C.) with cultigen means

Table 1. List of leafy Brassica oleracea cultigens and sources of seed.

\begin{tabular}{|c|c|c|c|c|}
\hline Cultigen & $\begin{array}{c}\text { B. oleracea } \\
\text { var. }\end{array}$ & Accession lot & Seed source/origin ${ }^{z}$ & Source location \\
\hline Bona & acephala & & Johnny's Selected Seeds & Albion, Maine \\
\hline Calvolo Palmizio Nero & selenesia & G 30714 92GI1 & USDA-ARS/Italy & Geneva, N.Y. \\
\hline Condor & mendolsa & G 30859 97GIU & USDA-ARS & Geneva, N.Y. \\
\hline Couve Espanhola & costata & G 29806 91GI1 & USDA-ARS & ieneva, N.Y. \\
\hline Couve Nabica & costata & 30855 96GI1 & USDA-ARS/Portugal & eneva, N.Y. \\
\hline Couve Portugueasa & costata & 2GI & USDA-ARS & Geneva, N.Y. \\
\hline Crimson Garden & acephala & & Johnny's Selected Seeds & Albion, Maine \\
\hline Giant Je & palmifolia & G 30723 92GI & USDA-ARS/UK & Geneva, N.Y. \\
\hline NZ Thou & ramosa & 96GI & USDA-ARS & ieneva, N.Y. \\
\hline Panc & costata & G 3 & & a, N.Y. \\
\hline Prer & selenesia & G 30729 90UO & USL & Geneva, N.Y. \\
\hline Redbor F1 & acephala & & Johnny's Selected Seeds & Albion, Maine \\
\hline Red Russian & acephala & & Johnny's Selected Seeds & Albion, Maine \\
\hline Ro & alboglabra & G 3 & & eva, N.Y. \\
\hline e & selenesia & G 3 & & J.Y. \\
\hline & acephala & 6GI1 & & va, N.Y. \\
\hline Siber & selenesia & G 2922189 UO & USD & Geneva, N.Y. \\
\hline Tall Marrowstem & medullosa & G 30717 92GI & USDA-ARS/UK & Geneva, N.Y. \\
\hline & acephala & & & Albion, Maine \\
\hline Winterbor F1 & acephala & & Johnny's Selected Seeds & Albion, Maine \\
\hline $30343-93 \mathrm{G} 1$ & acephala & G 303 & $\mathrm{ARS} / \mathrm{UK}$ & Geneva, N.Y. \\
\hline $30661-92 \mathrm{G} 1$ & acephala & G $30661-92 \mathrm{G} 1$ & USDA-ARS/UK & Geneva, N.Y. \\
\hline $30665-96 \mathrm{G} 1$ & acephala & G30661 96GI1 & USDA-ARS/UK & Geneva, N.Y. \\
\hline
\end{tabular}

${ }^{2}$ Origins not listed are either United States or unknown.

Table 2. Mean values ${ }^{2}$ (mg per $100 \mathrm{~g}$ fresh weight) of lutein and $\beta$-carotene for 23 leafy Brassica oleracea cultigens over two growing seasons (YR1 and $Y R 2)$.

\begin{tabular}{|c|c|c|c|c|}
\hline \multirow[b]{2}{*}{ Cultigens } & \multicolumn{2}{|c|}{ Lutein } & \multicolumn{2}{|c|}{$\beta$-carotene } \\
\hline & $Y R 1$ & $Y R 2$ & $Y R 1$ & $Y R 2$ \\
\hline Bona & $6.96 \pm 1.41$ & $7.14 \pm 1.29$ & $4.77 \pm 0.27$ & $5.67 \pm 0.88$ \\
\hline Calvolo Palmizio Nero & $12.36 \pm 1.03$ & $13.33 \pm 1.81$ & $7.80 \pm 0.45$ & $8.07 \pm 1.61$ \\
\hline Condor & $6.28 \pm 0.65$ & $6.92 \pm 0.23$ & $4.26 \pm 0.52$ & $5.97 \pm 0.49$ \\
\hline Couve Espanhola & $5.64 \pm 0.24$ & $6.98 \pm 0.39$ & $4.39 \pm 0.42$ & $5.40 \pm 0.28$ \\
\hline Couve Nabica & $7.14 \pm 0.94$ & $8.97 \pm 0.70$ & $5.13 \pm 0.70$ & $7.33 \pm 0.82$ \\
\hline Couve Portugueasa & $5.31 \pm 0.79$ & $6.42 \pm 0.53$ & $4.28 \pm 0.12$ & $5.66 \pm 0.30$ \\
\hline Crimson Garden & $6.66 \pm 0.36$ & $8.71 \pm 2.93$ & $4.75 \pm 0.83$ & $5.54 \pm 0.03$ \\
\hline Giant Jersey Kale & $5.53 \pm 0.79$ & $8.89 \pm 0.68$ & $4.99 \pm 0.70$ & $7.35 \pm 0.46$ \\
\hline NZ Thousand Head & $6.41 \pm 0.25$ & $7.24 \pm 0.40$ & $4.73 \pm 0.43$ & $5.81 \pm 0.04$ \\
\hline Panca de Chaves & $4.91 \pm 0.25$ & $6.09 \pm 2.05$ & $3.49 \pm 0.26$ & $4.32 \pm 0.89$ \\
\hline Premier & $7.96 \pm 0.64$ & $9.07 \pm 0.59$ & $7.44 \pm 1.29$ & $7.67 \pm 0.31$ \\
\hline Redbor F1 & $6.52 \pm 0.63$ & $8.07 \pm 0.28$ & $5.46 \pm 0.13$ & $6.51 \pm 0.41$ \\
\hline Red Russian & $7.69 \pm 1.91$ & $6.91 \pm 1.26$ & $5.82 \pm 1.33$ & $5.78 \pm 1.13$ \\
\hline Round Leaf Kale & $5.98 \pm 0.36$ & $8.29 \pm 0.89$ & $4.66 \pm 0.95$ & $6.34 \pm 0.84$ \\
\hline S.C. Green Glaze & $6.07 \pm 0.02$ & $6.27 \pm 0.76$ & $4.73 \pm 0.70$ & $4.74 \pm 1.31$ \\
\hline Shetland & $4.94 \pm 0.34$ & $7.12 \pm 0.39$ & $3.49 \pm 0.44$ & $6.39 \pm 0.35$ \\
\hline Siberian Improved & $7.32 \pm 0.21$ & $8.88 \pm 1.25$ & $5.60 \pm 0.42$ & $7.24 \pm 1.16$ \\
\hline Tall Marrowstem & $6.94 \pm 2.02$ & $8.20 \pm 2.05$ & $5.20 \pm 0.98$ & $6.60 \pm 1.56$ \\
\hline Toscano & $11.52 \pm 0.88$ & $13.43 \pm 0.77$ & $10.00 \pm 0.41$ & $9.92 \pm 0.57$ \\
\hline Winterbor F1 & $7.71 \pm 1.11$ & $9.60 \pm 0.78$ & $5.33 \pm 0.64$ & $7.01 \pm 1.13$ \\
\hline $30343-93 \mathrm{G} 1$ & $4.84 \pm 0.46$ & $5.83 \pm 0.01$ & $3.82 \pm 1.04$ & $5.08 \pm 0.23$ \\
\hline $30661-92 \mathrm{G} 1$ & $5.82 \pm 0.34$ & $6.96 \pm 1.19$ & $4.59 \pm 0.67$ & $5.53 \pm 1.07$ \\
\hline $30665-96 \mathrm{G} 11$ & $6.09 \pm 1.04$ & $6.90 \pm 1.21$ & $4.71 \pm 0.60$ & $5.76 \pm 1.35$ \\
\hline Mean & 6.81 & 8.10 & 5.19 & 6.33 \\
\hline $\operatorname{LSD}_{0.05}{ }^{\mathrm{y}}$ & 1.47 & 1.92 & 1.63 & 1.42 \\
\hline $\operatorname{LSD}_{0.05}{ }^{x}$ & \multicolumn{2}{|c|}{1.21} & \multicolumn{2}{|c|}{1.08} \\
\hline
\end{tabular}

${ }^{2}$ Composition of leaf samples from three replications, 10 plants each \pm standard error.

${ }^{y}$ LSD for differences between cultivar means within year.

${ }^{\mathrm{x}} \mathrm{LSD}$ for differences between cultivar means between years.

separated by least significant difference (LSD) of 0.05 within and between growing seasons. A correlation matrix for cultigens within each season was calculated for all variables tested. To test differences in rank order among the cultigens between the two growing seasons, Spearman's rank correlations were calculated (Steel and Torrie, 1980).

\section{Results and Discussion}

Lutein accumulation differed among cultigens $(P<0.001)$ and year $(P<0.001)$, but not for the interaction of cultigen and year. Total lutein concentration among the cultigens ranged from a high of $13.43 \mathrm{~g} \mathrm{FW}$ ('Toscano') to a low of $4.84 \mathrm{mg} / 100 \mathrm{~g} \mathrm{FW}$ (30343-93G1; Table 2). Lutein accumulation was significantly higher $(P<0.001)$ during year 2 . During year 1 , lutein ranged from $12.36 \mathrm{mg} / 100 \mathrm{~g} \mathrm{FW}$ ('Calvolo Palmizio Nero') to $4.84 \mathrm{mg} / 100 \mathrm{~g}$ FW (30343-93G1). During year 2, 'Toscano' displayed the highest lutein accumulation (13.43 mg/100 g FW ) while the lowest was again measured in 30343-93G1 (5.83 mg/100 g FW ). Previously, Mercadante and Rodriguez- 
Amaya (1991) reported lutein + violaxanthin values in two field-grown kale cultivars in Brazil to range from 11.4 to $7.1 \mathrm{mg} / 100 \mathrm{~g}$ FW. Violaxanthin is a diepoxide xanthophyll that is converted to zeaxanthin by the enzyme violaxanthin deepoxidase under light stress (Havaux and Niyogi, 1999). It can be difficult to separate lutein, violaxanthin, and zeaxanthin analytically due to their structural similarities; therefore, many authors report them together (Khachik et al., 1991). Because violaxanthin and zeaxanthin occur in small quantities in green leafy vegetables, comparisons between studies that report these compounds combined with lutein can be approximated. Results from the current study are within reported ranges for lutein accumulation in other field grown leafy B. oleracea cultigens.

Rank order of lutein did not significantly change for the cultigens from year 1 to year 2 [Spearman's rank correlation $(r s)=0.71$; $P<0.001]$. Cultigens with the highest lutein accumulation were 'Cavolo Palmizio Nero', 'Toscano', 'WinterborF1', 'Premier', 'Siberian Improved', 'Couve Nabica', 'Crimson Garden', and 'Tall Marrowstem'. Cultigens with the lowest lutein accumulation were 30343-93G1, 'Penca de Chaves', 'Couve Portuguesa', 'Shetland', 'S.C. Green Glaze', 'Couve Espanhola', 30661-92G1, and 30665-96G11.

$\beta$-carotene accumulation differed among cultigens $(P<0.001)$ and year $(P<0.001)$, but not for the interaction of cultigen and year. Total $\beta$-carotene accumulation among the cultigens ranged from a high of $10.00 \mathrm{mg} / 100$ g FW ('Toscano') to a low of $3.82 \mathrm{mg} / 100 \mathrm{~g}$ FW (30343-93G1; Table 2). $\beta$-carotene accumulation was also significantly higher $(P<$ $0.001)$ during year 2 . During year $1, \beta$-carotene ranged from $10.00 \mathrm{mg} / 100 \mathrm{~g} \mathrm{FW}$ ('Toscano') to $3.82 \mathrm{mg} / 100 \mathrm{~g} \mathrm{FW}$ (30343-93G1). During year 2, 'Toscano' again displayed the highest $\beta$-carotene accumulation, while the lowest was measured in 'Panca de Chaves'. Müller(1997) detected $7.28 \mathrm{mg} \beta$-carotene $/ 100 \mathrm{~g} \mathrm{FW}$ in fieldgrown kale of unknown cultigen. $\beta$-carotene values in our study are similar to previously reported values for other field-grown leafy $B$. oleracea cultivars (Kurilich et al., 1999; Mercadante and Rodriguez-Amaya, 1991).

Rank order of $\beta$-carotene also did not significantly change for the cultigens from year 1 to year 2 [Spearman's rank correlation $(r s)$ $=0.74 ; P<0.001]$. Cultigens with the highest $\beta$-carotene accumulation were 'Toscano', 'Cavolo Palmizio Nero', 'Premier', 'Siberian Improved', 'Couve Nabica', 'Giant Jersey Kale', and 'Redbor'. Cultigens with the lowest $\beta$-carotene accumulation were 'Penca de Chaves', 30343-93G1, 'S.C. Green Glaze', 'Couve Espanhola', 'Shetland', 'Couve Portuguesa', 30665-96G11+, and 'Condor'.

Chlorophyll $a$ content differed among cultigens $(P<0.001)$, year $(P<0.001)$, and for the interaction of cultigen and year $(P=$ $0.002)$. Chlorophyll $b$ content differed among cultigens $(P<0.001)$, year $(P<0.001)$, and for the interaction of cultigen and year $(P<$ 0.001). Chlorophyll $a+\mathrm{Chl} b$ also differed among the cultigens $(P<0.001)$, year $(P<$ $0.001)$, and for the interaction of cultigen and year $(P<0.001)$. Values for $\mathrm{Chl} a$ and $\mathrm{Chl} b$ were within previously reported ranges for kale (Table 3; Khachik et al., 1986). Rank order of Chl $a$, Chl $b$, and Chl $a+\mathrm{Chl} b$ did not significantly change for the cultigens from year 1 to year 2 [Spearman's rank correlation $(r s)=0.47, P=0.01 ;(r s)=0.72, P<0.001$; $(r s)=0.56, P=0.003$, respectively].

During each season, there were high correlations between carotenoid and chlorophyll accumulations (Table 4). Similarities in behavior of carotenoids and chlorophylls have been reported for other crop species (Grunwald et al., 1977; Terry and Abadía, 1986; ). Ihl et al. (1994) found chlorophylls to highly correlate with total carotenoid levels in the leaves of Swiss chard (Beta vulgaris L.). Our results support these correlative relationships among these $B$. oleracea cultigens (Table 4). This suggests it may be possible to use chlorophyll content, or green coloration, to estimate gross lutein and $\beta$-carotene concentration in leafy green vegetables.

There appears to be a strong genetic influence on carotenoid accumulation within several vegetable crop species. Kurilich et al. (1999) determined that $79 \%$ of $\beta$-carotene and $82 \%$ of $\alpha$-tocopherol variation in broccoli heads was due to genetic differences among 50 accessions. Kurilich and Juvik (1999) found significant differences for lutein, zeaxanthin, $\beta$-cyrptoxanthin, $\alpha$-carotene, $\beta$-carotene, and $\alpha$-tocopherol kernel content among 44 sweet and dent corn lines. Significant differences for lutein, $\beta$-carotene, and $\alpha$-tocopherol accumulation have been observed among several red pepper (Capsicum annuum L.) cultivars (Almela et al., 1991; Daood et al., 1996). However, few studies to date have investigated the lutein and $\beta$-carotene variability among leafy $B$.oleracea cultigens, which consistently rank highest for lutein and $\beta$-carotene accumulation (Heinonen et al., 1989; Holden et al., 1999).

Along with genetic factors, there also appears to be an environmental influence on carotenoid accumulation. Mercadante and

Table 3. Mean values ${ }^{z}$ (mg per $100 \mathrm{~g}$ fresh weight) of chlorophyll (Chl) $a$ and $b$ for 23 leafy Brassica oleracea cultigens over two growing seasons (YR1 and $Y R 2)$.

\begin{tabular}{|c|c|c|c|c|c|c|}
\hline \multirow[b]{2}{*}{ Cultigens } & \multicolumn{2}{|c|}{ Chl $a$} & \multicolumn{2}{|c|}{ Chl $b$} & \multicolumn{2}{|c|}{ Chl $a+$ Chl $b$} \\
\hline & $Y R 1$ & $Y R 2$ & YR1 & $Y R 2$ & YR1 & $Y R 2$ \\
\hline Bona & $137.93 \pm 11.39$ & $128.47 \pm 29.50$ & $41.26 \pm 1.10$ & $30.23 \pm 5.88$ & $179.19 \pm 11.40$ & $158.70 \pm 35.38$ \\
\hline Calvolo Palmizio Nero & $278.03 \pm 23.54$ & $202.86 \pm 21.76$ & $89.52 \pm 1.65$ & $58.50 \pm 5.60$ & $367.55 \pm 23.96$ & $261.36 \pm 26.74$ \\
\hline Condor & $140.92 \pm 32.23$ & $121.81 \pm 18.00$ & $39.19 \pm 6.15$ & $28.29 \pm 0.74$ & $180.11 \pm 38.34$ & $150.10 \pm 18.72$ \\
\hline Couve Espanhola & $157.05 \pm 4.97$ & $114.81 \pm 11.90$ & $40.10 \pm 4.72$ & $28.62 \pm 2.57$ & $197.15 \pm 9.69$ & $143.43 \pm 13.70$ \\
\hline Couve Nabica & $120.84 \pm 31.06$ & $131.01 \pm 19.15$ & $38.02 \pm 4.58$ & $32.82 \pm 2.67$ & $158.86 \pm 35.43$ & $163.83 \pm 21.73$ \\
\hline Couve Portugueasa & $126.12 \pm 26.58$ & $114.93 \pm 8.62$ & $38.09 \pm 5.28$ & $26.42 \pm 2.90$ & $164.21 \pm 31.61$ & $141.35 \pm 11.51$ \\
\hline Crimson Garden & $165.88 \pm 20.29$ & $143.61 \pm 32.40$ & $49.41 \pm 4.56$ & $39.35 \pm 6.65$ & $215.29 \pm 24.21$ & $182.96 \pm 38.99$ \\
\hline Giant Jersey Kale & $129.28 \pm 32.15$ & $151.92 \pm 5.97$ & $39.31 \pm 5.64$ & $35.63 \pm 1.31$ & $168.59 \pm 37.67$ & $187.55 \pm 6.39$ \\
\hline NZ Thousand Head & $118.99 \pm 8.80$ & $116.84 \pm 6.07$ & $35.78 \pm 0.96$ & $27.44 \pm 1.10$ & $154.77 \pm 9.76$ & $144.28 \pm 6.58$ \\
\hline Panca de Chaves & $123.24 \pm 9.83$ & $84.26 \pm 18.19$ & $32.13 \pm 4.17$ & $19.78 \pm 2.72$ & $155.37 \pm 12.54$ & $104.04 \pm 20.88$ \\
\hline Premier & $175.77 \pm 47.27$ & $148.71 \pm 16.82$ & $50.67 \pm 12.99$ & $37.34 \pm 3.82$ & $226.44 \pm 60.26$ & $186.05 \pm 20.21$ \\
\hline Redbor F1 & $161.53 \pm 13.70$ & $133.51 \pm 7.02$ & $46.60 \pm 3.17$ & $32.30 \pm 2.10$ & $208.13 \pm 16.70$ & $165.81 \pm 9.11$ \\
\hline Red Russian & $166.24 \pm 26.83$ & $113.69 \pm 21.49$ & $45.09 \pm 13.17$ & $28.25 \pm 5.51$ & $211.33 \pm 36.80$ & $141.94 \pm 26.93$ \\
\hline Round Leaf Kale & $128.62 \pm 18.98$ & $135.38 \pm 19.20$ & $38.82 \pm 0.90$ & $32.48 \pm 3.99$ & $167.44 \pm 18.84$ & $167.86 \pm 23.19$ \\
\hline S.C. Green Glaze & $146.17 \pm 8.32$ & $105.31 \pm 16.04$ & $41.04 \pm 0.78$ & $26.51 \pm 3.53$ & $187.21 \pm 7.55$ & $131.82 \pm 19.57$ \\
\hline Shetland & $109.77 \pm 11.42$ & $132.41 \pm 3.30$ & $30.95 \pm 1.21$ & $30.56 \pm 1.44$ & $140.72 \pm 11.63$ & $162.97 \pm 4.52$ \\
\hline Siberian Improved & $143.83 \pm 28.76$ & $145.21 \pm 6.87$ & $49.90 \pm 2.56$ & $37.36 \pm 1.76$ & $193.73 \pm 31.21$ & $182.57 \pm 7.61$ \\
\hline Tall Marrowstem & $170.52 \pm 18.96$ & $145.46 \pm 32.23$ & $40.27 \pm 7.47$ & $33.12 \pm 7.60$ & $210.79 \pm 21.75$ & $178.58 \pm 39.82$ \\
\hline Toscano & $276.00 \pm 40.84$ & $207.00 \pm 25.53$ & $86.82 \pm 5.80$ & $54.92 \pm 5.97$ & $362.82 \pm 46.32$ & $261.92 \pm 31.41$ \\
\hline Winterbor F1 & $164.26 \pm 28.02$ & $170.66 \pm 12.19$ & $49.60 \pm 9.39$ & $39.57 \pm 3.01$ & $213.86 \pm 37.11$ & $210.23 \pm 10.02$ \\
\hline 30343-93G1 & $144.60 \pm 14.46$ & $106.31 \pm 12.80$ & $34.23 \pm 3.90$ & $24.11 \pm 1.64$ & $178.83 \pm 14.36$ & $130.42 \pm 14.44$ \\
\hline 30661-92G1 & $134.43 \pm 14.76$ & $96.48 \pm 18.17$ & $37.80 \pm 1.11$ & $26.59 \pm 4.32$ & $172.23 \pm 15.37$ & $123.07 \pm 22.29$ \\
\hline 30665-96G11 & $157.61 \pm 21.07$ & $116.98 \pm 16.82$ & $42.86 \pm 7.05$ & $28.36 \pm 4.36$ & $200.47 \pm 28.11$ & $145.34 \pm 20.96$ \\
\hline Mean & 155.55 & 132.20 & 45.12 & 32.98 & 200.66 & 165.18 \\
\hline $\operatorname{LSD}_{0.05^{y}}{ }^{\mathrm{y}}$ & 40.17 & 28.72 & 9.64 & 6.44 & 46.98 & 36.24 \\
\hline $\operatorname{LSD}_{0.05}{ }^{x}$ & \multicolumn{2}{|c|}{24.22} & \multicolumn{2}{|c|}{5.64} & \multicolumn{2}{|c|}{29.27} \\
\hline
\end{tabular}

${ }^{\mathrm{z}}$ Composition of leaf samples from three replications, 10 plants each \pm standard error.

${ }^{y}$ LSD for differences between cultivar means within year.

${ }^{\mathrm{x}} \mathrm{LSD}$ for differences between cultivar means between years. 
Breeding, Cultivars, Rootstocks, \& Germplasm Resources

Table 4. Correlation coefficients ( $r$ ) between concentrations of carotenoids and chlorophylls (Chl) in leafy Brassica oleracea subspecies averaged across 23 cultigens over two growing seasons.

\begin{tabular}{|c|c|c|c|c|c|}
\hline Variable & Lutein & $\beta$-carotene & Chl $a$ & Chl $b$ & Chl $a+$ Chl $b$ \\
\hline \multicolumn{6}{|c|}{ YR 1: Correlation coefficients ( $r$ ) } \\
\hline Lutein & --- & $0.80^{* * * *}$ & $0.59^{* * * *}$ & $0.83^{* * *}$ & $0.67^{* * *}$ \\
\hline$\beta$-carotene & & --- & $0.62^{* * * *}$ & $0.74^{* * *}$ & $0.67^{* * * *}$ \\
\hline Chl $a$ & & & --- & $0.77^{* * * *}$ & $0.98^{* * *}$ \\
\hline Chl $b$ & & & & --- & $0.86^{* * *}$ \\
\hline Chl $a+$ Chl $b$ & & & & & --- \\
\hline \multicolumn{6}{|c|}{ YR 2: Correlation coefficients $(r)$} \\
\hline Lutein & --- & $0.86^{* * * *}$ & $0.85^{* * *}$ & $0.90^{* * *}$ & $0.87^{* * *}$ \\
\hline$\beta$-carotene & & --- & $0.79^{* * * *}$ & $0.82^{* * * *}$ & $0.80^{* * *}$ \\
\hline Chl $a$ & & & --- & $0.93^{* * *}$ & $0.99^{* * *}$ \\
\hline Chl $b$ & & & & --- & $0.96^{* * *}$ \\
\hline Chl $a+\operatorname{Chl} b$ & & & & & --- \\
\hline
\end{tabular}

Rodriguez-Amaya (1991) reported seasonal variation between winter and summer production in lutein and $\beta$-carotene levels between 'Manteiga' and 'Tronchuda' kale cultivars grown in Brazil. Results from the current study indicate a year-to-year variation in carotenoid accumulation as well. Although the cultigens were grown at exactly the same time of year during season 1 and season 2 , mean lutein and $\beta$-carotene values were higher during year 2 (Table 2). Despite these yearly variations, rank order of the cultigens for carotenoid accumulation did not change. Selecting cultivars that consistently rank high for carotenoid accumulation should allow producers to provide maximum carotenoid content for a given production year.

Assessing the genetic variability for carotenoid accumulation and identifying vegetable cultigens with maximum levels may have important health implications for consumers (Kurilich and Juvik, 1999). Recent scientific evidence describing the protective functions of lutein in the macular region of the eye has created interest in this carotenoid (Khachik et. al., 1997; Olsen, 1999; Snodderly, 1995). Dietary intake of lutein and $\beta$-carotene is associated with decreased risks of cancer and age-related macular degeneration (Mortensen et al., 2001). Studies indicate that a high intake of a variety of vegetables, providing a mixture of carotenoids, was more strongly associated with reduced cancer and eye disease risk than intake of individual carotenoid supplements (Johnson et al., 2000; Le Manchand et al., 1993). The B. oleracea germplasm grown in this study exhibited a 2.4 -fold difference in lutein and a 2.5 -fold difference in $\beta$-carotene concentration. Therefore, identifying $B$. oleracea cultigens with the genetic potential for high carotenoid accumulation may hold dietary and nutritional advantages.

Significant variability existed for lutein and $\beta$-carotene accumulation among 23 leafy $B$. oleracea cultigens grown in the northeastern region of the United States. The carotenoid content of the B. oleracea cultigens demonstrated a year-to-year variation as well. However, rank order of the carotenoid content of the cultigens did not change between the two seasons of production. The B. oleracea cultigens that consistently ranked highest for carotenoid accumulation were 'Calvolo
Palmizio Nero', 'Toscano', 'Premier', and 'Siberian Improved'. Under similar growing conditions, choice of $B$. oleracea cultigen will influence carotenoid accumulation, and this may affect the health benefits of consuming these leafy green vegetables.

Almela, L., J.M. López-Roca, M.E. Candela, and M.D. Alcázar. 1991. Carotenoid composition of new cultivars of red pepper for paprika. J. Agric. Food Chem. 39:1606-1609.

Balentine, D.A., M.C. Albano, and M.G. Nair. 1999. Role of medicinal plants, herbs, and spices in protecting human health. Nutr. Rev. 57(9):S41-S45.

Daood, H.G., M. Vinkler, F. Márkus, E.A. Hebshi, and P.A. Biacs. 1996. Antioxidant vitamin content of spice red pepper (paprika) as affected by technological and varietal factors. Food Chem. 55(4):365-372.

Goldman, I.L., A.A. Kader, and C.H. Heintz. 1999. Influence of production, handling, and storage on phytonutrient content of foods. Nutr. Rev. 57(9): S46-S52.

Grunwald, C., J.L. Sims, and S.J. Sheen. 1977. Effects of nitrogen fertilization and stalk position on chlorophyll, carotenoids, and certain lipids of three tobacco genotypes. Can. J. Plant Sci. 57:525-535.

Grusak, M.A., D. DellaPenna, and R.M. Welch. 1999. Physiological processes affecting the content and distribution of phytonutrients in plants. Nutr. Rev. 57(9):S27-S33.

Havaux, M. and K.K. Niyogi. 1999. The violaxanthin cycle protects plants from photooxidative damage by more than one mechanism. Proc. Natl. Acad. Sci. 96:8762-8767.

Heinonen, M.I., V. Ollilainen, E.K. Linkola, P.T. Varo, and P.E. Koivistoinen. 1989. Carotenoids in finnish foods: Vegetables, fruits, and berries. J. Agr. Food Chem. 37:655-659.

Holden, J.M., A.L. Eldridge, G.R. Beecher, I.M. Buzzard, S. Bhagwat, C.S. Davis, L.W. Douglass, S. Gebhardt, D. Haytowitz, and S. Schakel. 1999. Carotenoid content of U.S. foods: An update of the database. J. Food Comp. Anal. 12:169-196.

Howell, J.C., A.R. Bonnano, T. Jude Boucher, D.N. Ferro, and R.L. Wick (eds.). 2002. Broccoli, cabbage, cauliflower, and minor cole crops, p. 64-70. In: 2002-2003 New England vegetable management guide. UMass Ext. Offic. Commun. Mktg.

Ihl, M., C. Shene, E. Scheuermann, and V. Bifani. 1994. Correlation for pigment content through colour determination using tristimulus values in a green leafy vegetable, swiss chard. J. Sci. Food Agr. 66:527-531

Johnson, E.J., B.R. Hammond, K.J. Yeum, J. Qin, X.D. Wang, C. Castaneda, D.M. Snodderly, and R.M. Russell. 2000. Relation among serum and

\section{Literature Cited}

tissue concentrations of lutein and zeaxanthin and macular pigment density. Amer. J. Clinical Nutr. 71:1555-1562.

Jones, J.B., Jr. 1972. Plant tissues analysis for micronutrients, p. 319-346. In: J.J. Mortvedt, P.M. Giordano, and W.L. Lindsay (eds.). Micronutrients in agriculture. Soil Sci. Soc. Amer., Madison, Wis.

Khachik, F., G.R. Beecher, and N.F. Whittaker. 1986. Separation, identification, and quantification of the major carotenoid and chlorophyll constituents in extracts of several green vegetables by liquid chromatography. J. Agr. Food Chem. 34(4):603-616.

Khachik,F., G.R. Beecher, M.B. Goli, and W.R. Lusby. 1991. Separation, identification, and quantification of carotenoids in fruits, vegetables and human plasma by high performance liquid chromatography. Pure Appl. Chem. 63(1):71-80.

Khachik, F., P.S. Bernstein, and D.L. Garland. 1997. Identification of lutein and zeaxanthin oxidation products in human and monkey retinas. Investigative Ophthalmol. and Visual Sci. 38(9):1082-1811.

Kimura, M., D.B. Rodriguez-Amaya, and H.T. Godoy. 1990. Assessment of the saponification step in the quantification determination of carotenoids and provitamins A. Food Chem. 35:187-195.

Klein, B.P. and A.K. Perry. 1982. Ascorbic acid and vitamin A activity in selected vegetables from different geographic areas of the United States. J. Food Sci. 47:941-948.

Krinsky, N.I. 2002. Possible biologic mechanisms for a protective role of xanthophylls. J. Nutr. 132:540S-542S.

Kurilich, A.C., G.J. Tsau, A. Brown, L. Howard, B.P. Klein, E.H. Jeffery, M. Kushad, M.A. Walig, and J.A. Juvik. 1999. Carotene, tocopherol, and ascorbate in subspecies of Brassica oleracea. J. Agr. Food Chem. 47:1576-1581.

Kurilich, A.C. and J.A. Juvik. 1999. Quantification of carotenoid and tocopherol antioxidants in Zea mays. J. Agr. Food Chem. 47:1948-1955.

Le Marchand, L., J.H. Hankin, L.N. Kolonel, G.R. Beecher,L.R. Wilkens, and L.P.Zhao. 1993. Intake of specific carotenoids and lung cancer risk. Cancer Epidemiol. Biomarkers Prev. 2:183-187.

Mercadante, A.Z. and D.B. Rodriguez-Amaya. 1991. Carotenoid composition of a leafy vegetable in relation to some agricultural variables. J. Agr. Food Chem. 39(6):1094-1097.

Mortensen, A., L.H. Skibsted, and T.G. Truscott. 2001. The interaction of dietary carotenoids with radical species. Arch. Biochem. Biophys. 385(1):13-19.

Müller, H. 1997. Determination of the carotenoid content in selected vegetables and fruit by HPLC and photodiode array detection. ZLebensm Unters Forsch A 204:88-94.

Olsen, J.A. 1999. Carotenoids, p. 525 -541. In:M.E. Shils, J.A., Olsen, M. Shike, and A.C. Ross (eds.). Modern nutrition in health and disease. 9th ed. Williams and Wilkins, Baltimore.

Snodderly, D.M. 1995. Evidence for protection against age-related macular degeneration by carotenoids and antioxidant vitamins. Amer. J. Clinical Nutr. 62 (suppl.): 1448S-1461S.

Sommerburg, O., J.E.E. Keunen, A.C. Bird, and F.J.G.M. van Kuijk. 1998. Fruits and vegetables that are sources for lutein and zeaxanthin: The macular pigment in human eyes. Brit. J. Ophthalmol. 82(8):907-910.

Steel, R.G.D. and J.H. Torrie. 1980. Principles and procedures of statistics a biometrical approach. McGraw-Hill, New York.

Terry, N. and J. Abadía. 1986. Function of iron in chloroplasts. J. Plant Nutr. 9(3-7):609-646.

Tracewell, C.A., J.S. Vrettos, J.A. Bautista, H.A. Frank, and G.W. Brudvig. 2001. Carotenoid photooxidation in photosystem II. Arch. Biochem. Biophys. 385(1):61-69.

Zaripheh, S. and J.W. Erdman, Jr. 2002. Factors that influence the bioavailability of xanthophylls. J. Nutr. 132:531S-534S. 